\title{
On Estimation of Population Mean Using Information on Auxiliary Attribute
}

\author{
Rajesh Singh \\ Department of Statistics \\ BHU, Varanasi (U.P.), India \\ rsinghstat@yahoo.com
}

\begin{abstract}
We consider the problem of estimating the finite population mean when some information on auxiliary attribute is available. We obtain the mean square error (MSE) equation for the proposed estimators. It has been shown that the proposed estimator is better than Naik and Gupta (1996), Singh et al. (2008), AbdElfattah (2010) estimators. The results have been illustrated numerically by taking some empirical population considered in the literature.
\end{abstract}

Keywords: SRSWOR, Attribute, Point bi-serial correlation, MSE, Efficiency.

\section{Introduction}

In survey sampling the use of auxiliary information can increase the precision of an estimator when study variable $\mathrm{y}$ is highly correlated with the auxiliary variable $\mathrm{x}$. But in several practical situations, instead of existence of auxiliary variables there exists some auxiliary attributes, which are highly correlated with study variable y, such as (i) use of drugs and gender (ii) amount of milk produced and a particular breed of cow.

Consider a sample of size $\mathrm{n}$ drawn by simple random sampling without replacement (SRSWOR) from a population of size $\mathrm{N}$. Let $\mathrm{y}_{\mathrm{i}}$ and $\phi_{\mathrm{i}}$ denote the observations on variable $y$ and $\phi$ respectively for the $\mathrm{i}^{\text {th }}$ unit $(\mathrm{i}=1,2,3 \ldots . \mathrm{N}$.). It is assumed that attribute $\phi$ takes only the two values 0 and 1 according as

$\phi=1$, if $\mathrm{i}^{\text {th }}$ unit of the population possesses attribute $\phi$

$=0$, if otherwise.

Let $\mathrm{A}=\sum_{\mathrm{i}=1}^{\mathrm{N}} \phi_{\mathrm{i}}$ and $\mathrm{a}=\sum_{\mathrm{i}=1}^{\mathrm{n}} \phi_{\mathrm{i}}$ denote the total number of units in the population and sample possessing attribute $\phi$ respectively, $\mathrm{p}=\frac{\mathrm{A}}{\mathrm{N}}$ and $\mathrm{p}=\frac{\mathrm{a}}{\mathrm{n}}$ denote the proportion of units in the population and sample, respectively, possessing attribute $\phi$.

Define,

$\mathrm{e}_{\mathrm{y}}=\frac{(\overline{\mathrm{y}}-\overline{\mathrm{Y}})}{\overline{\mathrm{Y}}} \quad \mathrm{e}_{\phi}=\frac{(\mathrm{p}-\mathrm{P})}{\mathrm{P}}$,

$\mathrm{E}\left(\mathrm{e}_{\mathrm{i}}\right)=0,(\mathrm{i}=\mathrm{y}, \phi)$

$E\left(e_{y}^{2}\right)=f C_{y}^{2}, E\left(e_{\phi}^{2}\right)=f C_{p}^{2}, E\left(e_{y} e_{\phi}\right)=f \rho_{p b} C_{y} C_{p}$. 
Where

$$
\mathrm{f}=\left(\frac{1}{\mathrm{n}}-\frac{1}{\mathrm{~N}}\right) \quad \mathrm{C}_{\mathrm{y}}^{2}=\frac{\mathrm{S}_{\mathrm{y}}^{2}}{\overline{\mathrm{Y}}^{2}}, \quad \mathrm{C}_{\mathrm{p}}^{2}=\frac{\mathrm{S}_{\mathrm{p}}^{2}}{\mathrm{P}^{2}},
$$

and $\rho_{\mathrm{pb}}=\frac{\mathrm{S}_{\mathrm{y} \phi}}{\mathrm{S}_{\mathrm{y}} \mathrm{S}_{\phi}}$ is the point biserial correlation coefficient.

Here,

$\mathrm{S}_{\mathrm{y}}^{2}=\frac{1}{\mathrm{~N}-1} \sum_{\mathrm{i}=1}^{\mathrm{N}}\left(\mathrm{y}_{\mathrm{i}}-\overline{\mathrm{Y}}\right)^{2}, \quad \mathrm{~S}_{\phi}^{2}=\frac{1}{\mathrm{~N}-1} \sum_{\mathrm{i}=1}^{\mathrm{N}}\left(\phi_{\mathrm{i}}-\mathrm{P}\right)^{2} \quad$ and

$\mathrm{S}_{\mathrm{y} \phi}=\frac{1}{\mathrm{~N}-1}\left(\sum_{\mathrm{i}=1}^{\mathrm{N}} \mathrm{y}_{\mathrm{i}} \phi_{\mathrm{i}}-\mathrm{NP} \overline{\mathrm{Y}}\right)$

In order to have an estimate of the population mean $\bar{Y}$ of the study variable $y$, assuming the knowledge of the population proportion p, Naik and Gupta (1996) defined following ratio and product estimators

$$
\begin{aligned}
& \mathrm{t}_{\mathrm{NGR}}=\overline{\mathrm{y}}\left(\frac{\mathrm{P}}{\mathrm{p}}\right) \\
& \mathrm{t}_{\mathrm{NGP}}=\overline{\mathrm{y}}\left(\frac{\mathrm{p}}{\mathrm{P}}\right)
\end{aligned}
$$

The mean square error (MSE) of $t_{\text {NGR }}$ and $t_{\text {NGP }}$ up to the first order of approximation, respectively, are

$$
\begin{aligned}
& \operatorname{MSE}\left(\mathrm{t}_{\mathrm{NGR}}\right)=\mathrm{f} \overline{\mathrm{Y}}^{2}\left\lfloor\mathrm{C}_{\mathrm{y}}^{2}+\mathrm{C}_{\mathrm{p}}^{2}-2 \rho_{\mathrm{pb}} \mathrm{C}_{\mathrm{y}} \mathrm{C}_{\mathrm{p}}\right\rfloor \\
& \operatorname{MSE}\left(\mathrm{t}_{\mathrm{NGP}}\right)=\mathrm{f} \overline{\mathrm{Y}}^{2}\left\lfloor\mathrm{C}_{\mathrm{y}}^{2}+\mathrm{C}_{\mathrm{p}}^{2}+2 \rho_{\mathrm{pb}} \mathrm{C}_{\mathrm{y}} \mathrm{C}_{\mathrm{p}}\right\rfloor
\end{aligned}
$$

\section{Other estimators}

Singh et al. (2008) suggested the following ratio estimator

$$
\mathrm{t}_{\mathrm{S}}=\frac{\overline{\mathrm{y}}+\mathrm{b}_{\phi}(\mathrm{P}-\mathrm{p})}{\left(\mathrm{m}_{1} \mathrm{p}+\mathrm{m}_{2}\right)}\left(\mathrm{m}_{1} \mathrm{P}+\mathrm{m}_{2}\right)
$$

where $m_{1}(\neq 0)$ and $m_{2}$ are either real numbers or the functions of the parameters of the attribute such as $C_{p}, \beta_{2}(\phi)$ and $\rho_{\mathrm{pb}}$.

In Singh et al. (2008), MSE equation of these ratio- type estimators were given by

$$
\operatorname{MSE}\left(\mathrm{t}_{\mathrm{S}}\right)=\mathrm{f}\left\lfloor\operatorname{RS}_{\phi}^{2}+\mathrm{S}_{\mathrm{y}}^{2}\left(1-\rho_{\mathrm{pb}}^{2}\right)\right\rfloor
$$

where $\mathrm{R}$ depends on the choice of the parameters. 
Abd-Elfattah et al. (2010) proposed some ratio type estimators. The minimum MSE attained in Abd-Elfattah et al. (2010) was

$\operatorname{MSE}_{\text {min }}\left(t_{A b d}\right)=f\left[S_{y}^{2}\left(1-\rho_{p b}^{2}\right)\right]$

The minimum MSE of $t_{A b d}$ is equal to the MSE of regression estimator $\mathrm{t}_{\text {reg }}=\overline{\mathrm{y}}+\hat{\beta}(\mathrm{P}-\mathrm{p})$.

$\left(\operatorname{MSE}\left(t_{r e g}\right)=f\left[S_{y}^{2}\left(1-\rho_{\mathrm{pb}}^{2}\right)\right]\right)$.

Shabbir and Gupta (2007) considered following estimator

$\mathrm{t}_{\mathrm{SG}}=\overline{\mathrm{y}}\left[\mathrm{d}_{1}+\mathrm{d}_{2}(\mathrm{p}-\mathrm{P})\right]\left(\frac{\mathrm{P}}{\mathrm{p}}\right)$

where $d_{1}$ and $d_{2}$ are constants and whose sum is not necessarily equal to one.

The optimum MSE reported by Shabbir and Gupta (2007) of $t_{S G}$ is

$\operatorname{MSE}\left(\mathrm{t}_{\mathrm{SG}}^{0}\right)=\frac{\mathrm{fS}_{\mathrm{y}}^{2}\left(1-\rho_{\mathrm{pb}}^{2}\right)}{1+\mathrm{fC}_{\mathrm{y}}^{2}\left(1-\rho_{\mathrm{pb}}^{2}\right)}$

Unfortunately the expression obtained by Shabbir and Gupta (2007) is incorrect.

The corrected MSE of $\mathrm{t}_{\mathrm{SG}}$ is given as-

$\operatorname{MSE}\left(\mathrm{t}_{\mathrm{SG}}\right)_{\min }=\left[\overline{\mathrm{Y}}^{2}-\frac{\Delta_{1} \Delta_{5}^{2}+\Delta_{3} \Delta_{4}^{2}-2 \Delta_{2} \Delta_{4} \Delta_{5}}{\Delta_{1} \Delta_{3}-\Delta_{2}^{2}}\right]$

where,

$$
\begin{aligned}
& \Delta_{1}=\overline{\mathrm{Y}}^{2}+\overline{\mathrm{Y}}^{2} \mathrm{f}\left(\mathrm{C}_{\mathrm{y}}^{2}+3 \mathrm{C}_{\mathrm{x}}^{2}-4 \rho \mathrm{C}_{\mathrm{y}} \mathrm{C}_{\mathrm{x}}\right), \quad \Delta_{2}=\overline{\mathrm{X}} \overline{\mathrm{Y}} \mathrm{f}\left(2 \mathrm{C}_{\mathrm{x}}^{2}-\rho \mathrm{C}_{\mathrm{y}} \mathrm{C}_{\mathrm{x}}\right) \\
& \Delta_{3}=\overline{\mathrm{X}}^{2} \mathrm{fC}_{\mathrm{x}}^{2}, \quad \Delta_{4}=\overline{\mathrm{Y}}^{2} \mathrm{f}\left(\mathrm{C}_{\mathrm{x}}^{2}-\rho \mathrm{C}_{\mathrm{y}} \mathrm{C}_{\mathrm{x}}\right)+\overline{\mathrm{Y}}^{2}, \quad \Delta_{5}=\overline{\mathrm{X}} \overline{\mathrm{Y}}_{\mathrm{x}}^{2} .
\end{aligned}
$$

\section{The proposed estimator}

We define a family of ratio estimators of population mean $\overline{\mathrm{Y}}$ as

$$
\mathrm{t}_{\alpha}=\alpha_{1} \overline{\mathrm{y}}+\alpha_{2} \overline{\mathrm{y}}\left(\frac{\mathrm{m}_{1} \mathrm{P}+\mathrm{m}_{2}}{\mathrm{~m}_{1} \mathrm{p}+\mathrm{m}_{2}}\right)^{\alpha}
$$

where $m_{1}$ and $m_{2}$ are same as defined in (2.2) and $\alpha_{1}$ and $\alpha_{2}$ are real constants to be determined such that the MSE of $t_{\alpha}$ is minimum.

Remark 1: Here we would like to mention that the choice of the estimator depends on the availability and values of the various parameter(s) used (for choice of the parameters $\mathrm{m}_{1}$ and $\mathrm{m}_{2}$ refer to Singh et al. (2008) and Singh and Kumar (2009)). 
Expressing $\mathrm{t}_{\alpha}$ in terms of e's we have

$$
\mathrm{t}_{\alpha}=\overline{\mathrm{Y}}\left[\alpha_{1}\left(1+\mathrm{e}_{\mathrm{y}}\right)+\alpha_{2}\left(1+\theta \mathrm{e}_{\phi}\right)-\alpha\right]
$$

where $\theta=\frac{\mathrm{aP}}{\mathrm{ap}+\mathrm{b}}$.

Expanding the right hand side of (3.2) and retaining terms up to second power of e's, we have

$$
\mathrm{t}_{\alpha}=\overline{\mathrm{Y}}\left[\alpha_{1}\left(1+\mathrm{e}_{\mathrm{y}}\right)+\alpha_{2}\left\{1-\alpha \theta \mathrm{e}_{\phi}+\frac{\alpha(\alpha+1)}{2} \theta^{2} \mathrm{e}_{\phi}^{2}+\mathrm{e}_{\mathrm{y}}-\alpha \theta \mathrm{e}_{\mathrm{y}} \mathrm{e}_{\phi}\right\}\right]
$$

Subtracting $\bar{Y}$ from both side of (3.3) and then taking expectations, we get the bias of the estimator $\mathrm{t}_{\alpha}$ up to the first order of approximation, as

$$
\mathrm{B}\left(\mathrm{t}_{\alpha}\right)=\overline{\mathrm{Y}}\left[\left(\alpha_{1}+\alpha_{2}-1\right)+\alpha_{2} \mathrm{f}\left\{\frac{\alpha(\alpha+1)}{2} \theta^{2} \mathrm{C}_{\mathrm{p}}^{2}-\alpha \theta \rho_{\phi} \mathrm{C}_{\mathrm{y}} \mathrm{C}_{\mathrm{p}}\right\}\right]
$$

Subtracting $\bar{Y}$ from both side of (3.3), squaring and then taking expectations, we get MSE of the estimator $t_{\alpha}$ up to the first order of approximation, as

$$
\operatorname{MSE}\left(\mathrm{t}_{\alpha}\right)=\overline{\mathrm{Y}}^{2}\left\lfloor\alpha_{1}^{2} \mathrm{~A}_{1}+\alpha_{2}^{2} \mathrm{~A}_{2}+2 \alpha_{1} \alpha_{2} \mathrm{~A}_{3}-2 \alpha_{2} \mathrm{~A}_{4}-2 \alpha_{1}+1\right\rfloor
$$

where

$$
\begin{aligned}
& \mathrm{A}_{1}=1+\mathrm{fC} \mathrm{C}_{\mathrm{y}}^{2} \\
& \mathrm{~A}_{2}=1+\mathrm{f}\left\{\mathrm{C}_{\mathrm{y}}^{2}+\alpha^{2} \theta^{2} \mathrm{C}_{\mathrm{p}}^{2}-4 \alpha \theta \rho_{\phi} \mathrm{C}_{\mathrm{yCp}}+\alpha(\alpha+1) \theta^{2} \mathrm{C}_{\mathrm{p}}^{2}\right\} \\
& \mathrm{A}_{3}=1+\mathrm{f}\left\{\frac{\alpha(\alpha+1)}{2} \theta^{2} \mathrm{C}_{\mathrm{p}}^{2}+\mathrm{C}_{\mathrm{y}}^{2}-2 \alpha \theta \rho_{\phi} \mathrm{C}_{\mathrm{y}} \mathrm{C}_{\mathrm{p}}\right\} \\
& \mathrm{A}_{4}=1+\mathrm{f}\left\{\frac{\alpha(\alpha+1)}{2} \theta^{2} \mathrm{C}_{\mathrm{p}}^{2}-\alpha \theta \rho_{\phi} \mathrm{C}_{\mathrm{y}} \mathrm{C}_{\mathrm{p}}\right\} \\
& \alpha_{1}^{*}=\frac{\mathrm{A}_{2}-\mathrm{A}_{3} \mathrm{~A}_{4}}{\mathrm{~A}_{1} \mathrm{~A}_{2}-\mathrm{A}_{3}^{2}} \text { and } \quad \alpha_{2}^{*}=\frac{\mathrm{A}_{1} \mathrm{~A}_{4}-\mathrm{A}_{3}}{\mathrm{~A}_{1} \mathrm{~A}_{2}-\mathrm{A}_{3}^{2}}
\end{aligned}
$$

Substituting these optimum values of $\alpha_{1}^{*}$ and $\alpha_{2}^{*}$ in (3.5), we get the minimum MSE of $\mathrm{t}_{\alpha}$ as-

$$
\operatorname{MSE}\left(\mathrm{t}_{\alpha}\right)_{\min }=\overline{\mathrm{Y}}^{2}\left[1-\frac{\mathrm{A}_{2}+\mathrm{A}_{1} \mathrm{~A}_{4}^{2}-2 \mathrm{~A}_{3} \mathrm{~A}_{4}}{\mathrm{~A}_{1} \mathrm{~A}_{2}-\mathrm{A}_{3}^{2}}\right]
$$




\section{Another estimator}

Singh et al. (2007) suggested exponential ratio type and exponential product type estimators, respectively, as

$$
\begin{aligned}
& \mathrm{t}_{\mathrm{SR}}=\overline{\mathrm{y}} \exp \left[\frac{\mathrm{P}-\mathrm{p}}{\mathrm{P}+\mathrm{p}}\right] \\
& \mathrm{t}_{\mathrm{SP}}=\overline{\mathrm{y}} \exp \left[\frac{\mathrm{p}-\mathrm{P}}{\mathrm{p}+\mathrm{P}}\right]
\end{aligned}
$$

MSE expressions for the estimators $\mathrm{t}_{\mathrm{SR}}$ and $\mathrm{t}_{\mathrm{SP}}$ are given, respectively, as

$$
\begin{aligned}
& \operatorname{MSE}\left(\mathrm{t}_{\mathrm{SR}}\right)=\mathrm{f} \overline{\mathrm{Y}}^{2}\left[\mathrm{C}_{\mathrm{y}}^{2}+\frac{\mathrm{C}_{\mathrm{p}}^{2}}{4}-\rho_{\phi} \mathrm{C}_{\mathrm{y}} \mathrm{C}_{\mathrm{p}}\right] \\
& \operatorname{MSE}\left(\mathrm{t}_{\mathrm{SP}}\right)=\mathrm{f} \overline{\mathrm{Y}}^{2}\left[\mathrm{C}_{\mathrm{y}}^{2}+\frac{\mathrm{C}_{\mathrm{p}}^{2}}{4}+\rho_{\phi} \mathrm{C}_{\mathrm{y}} \mathrm{C}_{\mathrm{p}}\right]
\end{aligned}
$$

Using (3.1) and Singh et al. (2007) estimator, we define another family of estimators for population mean $\overline{\mathrm{Y}}$ as

$\mathrm{t}_{\mathrm{w}}=\left\{\mathrm{w}_{1} \overline{\mathrm{y}}+\mathrm{w}_{2}(\mathrm{P}-\mathrm{p})\right\}\left\{\frac{\mathrm{aP}+\mathrm{b}}{\mathrm{ap}+\mathrm{b}}\right\}^{\alpha} \exp \left\{\frac{(\mathrm{aP}+\mathrm{b})-(\mathrm{ap}+\mathrm{b})}{(\mathrm{aP}+\mathrm{b})+(\mathrm{ap}+\mathrm{b})}\right\}^{\beta}$

where $\mathrm{w}_{1}$ and $\mathrm{w}_{2}$ are constants and whose sum is not necessarily equal to one.

The Bias and MSE expressions of $\mathrm{t}_{\mathrm{w}}$ are respectively, given by

$$
\begin{array}{r}
\operatorname{Bias}\left(\mathrm{t}_{\mathrm{p}}\right)=\left(\mathrm{w}_{1}-1\right) \overline{\mathrm{Y}}+\mathrm{f}\left[\left(\mathrm{w}_{1} \overline{\mathrm{Y}} \mathrm{A}+\mathrm{w}_{2} \mathrm{~PB}\right) \mathrm{C}_{\mathrm{x}}^{2}-\mathrm{w}_{1} \overline{\mathrm{Y}} \mathrm{B} \rho \mathrm{C}_{\mathrm{y}} \mathrm{C}_{\mathrm{x}}\right\rfloor \\
\operatorname{MSE}\left(\mathrm{t}_{\mathrm{p}}\right)=\left(\mathrm{w}_{1}-1\right)^{2} \overline{\mathrm{Y}}^{2}+\mathrm{w}_{1}^{2}\left(\mathrm{~m}_{1}+2 \mathrm{~m}_{3}\right)+\mathrm{w}_{2}^{2} \mathrm{~m}_{2} \\
+2 \mathrm{w}_{1} \mathrm{w}_{2}\left(-\mathrm{m}_{4}-\mathrm{m}_{5}\right)-2 \mathrm{w}_{1} \mathrm{~m}_{3}+2 \mathrm{w}_{2} \mathrm{~m}_{5}
\end{array}
$$

where,

$$
\begin{aligned}
& \mathrm{A}=\frac{\theta^{2}}{8}[4 \alpha(\alpha+1)+\beta(\beta+2)+4 \alpha \beta], \quad \mathrm{B}=\left(\alpha+\frac{\beta}{2}\right) \theta, \\
& \mathrm{m}_{1}=\overline{\mathrm{Y}}^{2} \mathrm{f}\left(\mathrm{C}_{\mathrm{y}}^{2}+\mathrm{B}^{2} \mathrm{C}_{\mathrm{x}}^{2}-2 \mathrm{~B} \rho \mathrm{C}_{\mathrm{y}} \mathrm{C}_{\mathrm{x}}\right), \quad \mathrm{m}_{2}=\overline{\mathrm{X}}^{2} \mathrm{f}\left(\mathrm{C}_{\mathrm{x}}^{2}\right), \\
& \mathrm{m}_{3}=\overline{\mathrm{Y}}^{2} \mathrm{f}\left(\mathrm{AC}_{\mathrm{x}}^{2}-2 \mathrm{~B} \rho \mathrm{C}_{\mathrm{y}} \mathrm{C}_{\mathrm{x}}\right), \quad \mathrm{m}_{4}=\overline{\mathrm{Y} X} \mathrm{f}\left(-\mathrm{BC}_{\mathrm{x}}^{2}+\rho \mathrm{C}_{\mathrm{y}} \mathrm{C}_{\mathrm{x}}\right) \text {, } \\
& \mathrm{m}_{5}=\overline{\mathrm{X}} \overline{\mathrm{Y}} \mathrm{f}\left(-\mathrm{BC}_{\mathrm{x}}^{2}\right)
\end{aligned}
$$


Differentiating equation (4.7) with respect to $\mathrm{w}_{1}$ and $\mathrm{w}_{2}$ and than equating to zero we get $\mathrm{w}_{1}^{*}=\frac{\mathrm{L}_{3} \mathrm{~L}_{4}-\mathrm{L}_{2} \mathrm{~L}_{5}}{\left(\mathrm{~L}_{1} \mathrm{~L}_{3}-\mathrm{L}_{2}^{2}\right)^{2}}$ and $\quad \mathrm{w}_{2}^{*}=\frac{\mathrm{L}_{1} \mathrm{~L}_{5}-\mathrm{L}_{2} \mathrm{~L}_{4}}{\left(\mathrm{~L}_{1} \mathrm{~L}_{3}-\mathrm{L}_{2}^{2}\right)^{2}}$

where
$\mathrm{L}_{1}=\left(\overline{\mathrm{Y}}^{2}+\mathrm{m}_{1}+2 \mathrm{~m}_{3}\right)$
$\mathrm{L}_{2}=\left(-\mathrm{m}_{4}-\mathrm{m}_{5}\right)$
$\mathrm{L}_{3}=\mathrm{m}_{2}$
$\mathrm{L}_{4}=\left(\mathrm{m}_{3}+\overline{\mathrm{Y}}^{2}\right)$
$\mathrm{L}_{5}=\left(-\mathrm{m}_{5}\right)$

Substituting these optimum values of $\mathrm{w}_{1}^{*}$ and $\mathrm{w}_{2}^{*}$ in (4.7), we get the minimum MSE of $\mathrm{t}_{\mathrm{p}}$ as-

$\operatorname{MSE}\left(\mathrm{t}_{\mathrm{p}}\right)_{\min }=\left[\overline{\mathrm{Y}}^{2}-\frac{\mathrm{L}_{1} \mathrm{~L}_{5}^{2}+\mathrm{L}_{3} \mathrm{~L}_{4}^{2}-2 \mathrm{~L}_{2} \mathrm{~L}_{4} \mathrm{~L}_{5}}{\mathrm{~L}_{1} \mathrm{~L}_{3}-\mathrm{L}_{2}^{2}}\right]$

\section{Efficiency comparison:}

First, we compare the efficiency of proposed estimator $t_{\alpha}$ with usual estimator and than with regression estimator.

The variance of the usual estimator $\bar{y}$ is given by

$$
\begin{gathered}
\mathrm{V}(\overline{\mathrm{y}})=\mathrm{fC}_{\mathrm{y}}^{2} \\
\operatorname{MSE}\left(\mathrm{t}_{\alpha}\right)_{\min } \leq \mathrm{V}(\overline{\mathrm{y}}) \\
{\left[1-\frac{\mathrm{A}_{2}+\mathrm{A}_{1} \mathrm{~A}_{4}^{2}-2 \mathrm{~A}_{3} \mathrm{~A}_{4}}{\mathrm{~A}_{1} \mathrm{~A}_{2}-\mathrm{A}_{3}^{2}}\right] \leq \overline{\mathrm{Y}}^{2} \mathrm{f}_{1} \mathrm{C}_{\mathrm{y}}^{2}}
\end{gathered}
$$

On solving, we observe that above condition always holds true. Therefore, proposed estimator $\mathrm{t}_{\alpha}$ under optimum condition performs better than usual estimator.

Similarly, it can be shown that

$$
\operatorname{MSE}\left(\mathrm{t}_{\alpha}\right)_{\min } \leq \operatorname{MSE}(\mathrm{reg})=\operatorname{MSE}_{\min }\left(\mathrm{t}_{\mathrm{Abd}}\right)
$$

If,

$$
\overline{\mathrm{Y}}^{2}\left[1-\frac{\mathrm{A}_{2}+\mathrm{A}_{1} \mathrm{~A}_{4}^{2}-2 \mathrm{~A}_{3} \mathrm{~A}_{4}}{\mathrm{~A}_{1} \mathrm{~A}_{2}-\mathrm{A}_{3}^{2}}\right] \leq \overline{\mathrm{Y}}^{2} \mathrm{f}_{1} \mathrm{C}_{\mathrm{y}}^{2}\left(1-\rho_{\phi}^{2}\right)
$$

This is also true for all values of $\alpha(-1,0,1)$. 
Next, we compare the efficiency of proposed estimator $t_{p}$ with usual estimator and than with regression estimator.

$$
\operatorname{MSE}\left(\mathrm{t}_{\mathrm{p}}\right)_{\min } \leq \mathrm{V}(\overline{\mathrm{y}})
$$

If,

$$
\left[\overline{\mathrm{Y}}^{2}-\frac{\mathrm{L}_{1} \mathrm{~L}_{5}^{2}+\mathrm{L}_{3} \mathrm{~L}_{4}^{2}-2 \mathrm{~L}_{2} \mathrm{~L}_{4} \mathrm{~L}_{5}}{\mathrm{~L}_{1} \mathrm{~L}_{3}-\mathrm{L}_{2}^{2}}\right] \leq \mathrm{f}_{1} \overline{\mathrm{Y}}^{2} \mathrm{C}_{\mathrm{y}}^{2}
$$

On simplification, we observe that above condition is always true. Therefore proposed estimator $\left(\mathrm{t}_{\mathrm{w}}\right)$ min performs better than usual estimator in all situations.

Similarly it can be shown that

$$
\operatorname{MSE}\left(\mathrm{t}_{\mathrm{p}}\right)_{\min } \leq \operatorname{MSE}(\mathrm{reg})=\operatorname{MSE}_{\min }\left(\mathrm{t}_{\text {Abd }}\right)
$$

If,

$$
\left[\overline{\mathrm{Y}}^{2}-\frac{\mathrm{L}_{1} \mathrm{~L}_{5}^{2}+\mathrm{L}_{3} \mathrm{~L}_{4}^{2}-2 \mathrm{~L}_{2} \mathrm{~L}_{4} \mathrm{~L}_{5}}{\mathrm{~L}_{1} \mathrm{~L}_{3}-\mathrm{L}_{2}^{2}}\right] \leq \overline{\mathrm{Y}}^{2} \mathrm{f}_{1} \mathrm{C}_{\mathrm{y}}^{2}\left(1-\rho_{\phi}^{2}\right)
$$

This is also true for all values of $\alpha(-1,0,1$. ) and $\beta(-1,0,1$.)

Finally we have compared the efficiency of proposed estimator $t_{w}$ with the estimator $t_{p}$ $\operatorname{MSE}\left(\mathrm{t}_{\mathrm{p}}\right)_{\min } \leq \operatorname{MSE}\left(\mathrm{t}_{\alpha}\right)_{\min }$

Or if,

$$
\left[\overline{\mathrm{Y}}^{2}-\frac{\mathrm{L}_{1} \mathrm{~L}_{5}^{2}+\mathrm{L}_{3} \mathrm{~L}_{4}^{2}-2 \mathrm{~L}_{2} \mathrm{~L}_{4} \mathrm{~L}_{5}}{\mathrm{~L}_{1} \mathrm{~L}_{3}-\mathrm{L}_{2}^{2}}\right] \leq \overline{\mathrm{Y}}^{2}\left[1-\frac{\mathrm{A}_{2}+\mathrm{A}_{1} \mathrm{~A}_{4}^{2}-2 \mathrm{~A}_{3} \mathrm{~A}_{4}}{\mathrm{~A}_{1} \mathrm{~A}_{2}-\mathrm{A}_{3}^{2}}\right]
$$

The conditions depends upon choice of $\alpha$ and $\beta$.

\section{Empirical study}

We have used the data given in Sukhatme and Sukhatme ((1970) p. 256). Where, $Y$ : Number of villages in the circle and

$\phi$ : represent A circle consisting more than five villages.

The following Table shows percent relative efficiencies (PRE's) of different estimator's with respect to usual estimator.

$\begin{array}{lllllll}\mathrm{n} & \mathrm{N} & \overline{\mathrm{Y}} & \mathrm{P} & \rho_{\mathrm{pb}} & \mathrm{Cy} & \mathrm{C}_{\mathrm{p}} \\ 23 & 89 & 1102 & 0.1236 & 0.643 & 0.65405 & 2.19012\end{array}$


Table 1: PRE of different estimators with respect to usual estimator

\begin{tabular}{|c|c|}
\hline Estimator & PRE \\
\hline$\overline{\mathrm{y}}$ & 100 \\
\hline $\mathrm{t}_{\mathrm{NGR}}$ & 12.648 \\
\hline $\mathrm{t}_{\mathrm{RER}}$ & 60.603 \\
\hline $\mathrm{t}_{\mathrm{s}(\mathrm{opt})}$ & 170.488 \\
\hline$\left(\mathrm{t}_{\mathrm{SG}}\right)_{\min }$ & 172.120 \\
\hline$\left(\mathrm{t}_{\alpha}\right)_{\min }$ & 173.132 \\
\hline $\mathrm{t}_{\mathrm{w}} \quad \alpha=1, \beta=0$ & 172.120 \\
$\alpha=0, \beta=1$ & 187.804 \\
$\alpha=1, \beta=1$ & $\mathbf{3 9 2 . 6 2}$ \\
\hline
\end{tabular}

\section{Conclusion}

From Table 1, one can see that the proposed estimator t $\alpha$ under optimum condition performs better than the Shabbir and Gupta (2007) estimator, Singh et al. (2008) estimator and usual estimator. Also, the performance of the second proposed estimator tw depends upon choice of $\alpha$ and $\beta$. For $\alpha=1, \beta=1$, it attains maximum efficiency.

\section{References}

1. Abd-Elfattah, A.M. El-Sherpieny, E.A. Mohamed, S.M. Abdou, O.F. (2010). Improvement in estimating the population mean in simple random sampling using information on auxiliary attribute. Applied Mathematics Computation, doi:10.1016/j.amc.2009.12.041.

2. Naik, V.D., Gupta, P.C. (1996). A note on estimation of mean with known population of an auxiliary character. Journal of Indian Society of Agricultural Statistics 48(2) 151-158.

3. Shabbir, J., Gupta, S. (2007). On estimating the finite population mean with known population proportion of an auxiliary variable. Pakistan Journal of Statistics 23 (1) 1-9.

4. Singh, R. Chauhan, P. Sawan, N. Smarandache, F. (2007). Ratio-product type exponential estimator for estimating finite population mean using information on auxiliary attribute. Renaissance High Press. 
5. Singh, R. Chauhan, P. Sawan, N. Smarandache, F. (2008). Ratio estimators in simple random sampling using information on auxiliary attribute. Pakistan journal of statistics and operations research 4 (1) 47-53

6. Singh, R., Kumar, M. and Smarandache, F. (2008). Almost unbiased estimator for estimating population mean using known value of some population parameter(s). Pakistan journal of statistics and operations research 4(2) pp63-76.

7. Singh, R. and Kumar, M. (2009). A note on transformations on auxiliary variable in survey sampling. Model Assisted Statistics and Applications (Accepted).

8. Sukhatme, P.V. and Sukhatme, B.V. (1970). Sampling theory of surveys with applications. Iowa State University Press, Ames, U.S.A. 